\title{
Lower Bounds on the Width of Stark-Wannier Type Resonances
}

\author{
J. Asch, P. Briet \\ Centre de Physique Théorique, CNRS - Luminy, Case 907, F-13288 Marseille Cedex 9, France. \\ E-mail: asch@cpt.univ-mrs.fr
}

Received: 1 November 1995/Accepted: 29 January 1996

Abstract: We prove that the Schrödinger operator $-d^{2} / d x^{2}+F x+W(x)$ on $L^{2}(\mathbf{R})$ with $W$ bounded and analytic in a strip has no resonances in a region $\operatorname{Im} E \geq-\exp (-C / F)$.

\section{Introduction and Main Result}

The resonance problem of a Schrödinger particle subject to an electric field with nonvanishing mean bears interesting physical and mathematical aspects and has attracted much activity in both fields. From the point of view of transport in solids not only fluctuations of short range or Coulomb type but also random, quasiperiodic and especially periodic potentials, the Wannier case [25], are of interest. For the physics we refer to Avron [3], Grecchi and Sacchetti [15] and their references. Mathematically the classical questions are definition, existence and location of resonances. They are non-trivial even in a one dimensional situation.

For the case of fluctuations which do not decay at infinity the definition setup for resonances by spectral deformation was essentially given by Herbst and Howland [18].

The existence of resonances in the Wannier case was discussed by Avron [3]. Rigorous results were obtained in the high field regime by Agler, Froese [1]; in a small field and semiclassical context by Combes and Hislop [10], Bentosela and Grecchi [5]; for potentials with a finite number of bands by Buslaev and Dmitrieva [9], Grecchi and Maioli and Sacchetti [14] who have also results for the disordered case with large periods [13]. The techniques in $[10,5,13,14]$ were spectral deformation and perturbation theory; in [9] the complex poles of the reflection coefficient were studied directly by ODE methods; in [1] a Birman-Schwinger technique was employed.

Concerning the location in the Wannier case it is suggested by the Zener tilted band picture that the width -or imaginary part, or the inverse lifetime of the resonancesis exponentially small in the strength of the homogeneous part of the field [3]. The works on the existence confirm this: upper bounds on the imaginary part were given in $[10,5,14]$ for the semiclassical and for the finite band case. 
There are several results concerning the notorious problem of lower bounds on the resonance width. In [4] Bentosela et al. proved for a very general class of fluctuations that the spectrum is purely absolutely continuous. In [9] asymptotics of the resonance width in the field strength were obtained by a detailed study of the wavefunctions in the Wannier case for finite band potentials; Jensen [20] showed for bounded analytic fluctuations that resonances go away from the real axis with increasing field strength; Ahia [2] gave an exponential bound with an explicit constant for compactly supported fluctuations.

Lower bounds on the resonance width of Schrödinger particles in potentials decaying at infinity are known from the works of Harrell [16] and of Fernandez, Lavine [11] for potentials of compact support and from Helffer, Sjöstrand [17] for harmonic wells on an island in the semiclassical limit. The results in $[11,17]$ apply in more than one dimension.

Our contribution are lower bounds which are exponentially small in the field strength; they are valid for the class of potentials for which they are expected to be true.

The condition on the potential is:

(A) Let $W$ be analytic and bounded in a strip around and real on the real axis.

The result is:

Theorem 1. Let $W$ satisfy (A). There are $F_{0}>0, c>0$ such that the selfadjoint operator

$$
-d^{2} / d x^{2}+F x+W
$$

uniquely defined by extension from $C_{0}^{\infty}(\mathbf{R})$, has no resonances in the region

$$
\left\{E \in \mathbf{C}, \operatorname{Im} E \geq-e^{-c / F}\right\} \quad\left(F<F_{0}\right) .
$$

Let us describe in prose the idea -which is promising to apply in other situationsand the organisation of the proof.

Resonances of the Hamiltonian $H=-\Delta+F x+W$ are defined as eigenvalues of the complex distorted $H(\theta)=U(\theta) H U^{-1}(\theta)$. The distortion $U(\theta) \psi(x):=(1+$ $\left.\theta f^{\prime}\right)^{1 / 2} \psi(x+\theta f(x))$ is well defined if $\sup \left|\theta f^{\prime}\right|<1$. $\mathrm{f}$ will be chosen according to the following observation.

From the relation $\left(1+\theta f^{\prime}\right)(H(\theta)-E)\left(1+\theta f^{\prime}\right)=H-E+\theta P f+O\left(\theta^{2}\right)$ with the differential expression $P$ defined by

$$
P f:=-\frac{f^{\prime \prime \prime}}{2}+2(F x+W-E) f^{\prime}+\left(F+W^{\prime}\right) f
$$

it will be deduced that the existence of an $f$ which satisfies $P f=-1$ and is slowly oscillating implies (for $\operatorname{Re} \theta=0$ ) that $\operatorname{Im}(H(\theta)-E$ ) is negative for $-\operatorname{Im} E-\operatorname{Im} \theta<$ 0 . So there are no resonances with $\operatorname{Im} E>-1 / \sup \left|f^{\prime}\right|$. The problem of bounding $\operatorname{ImE}$ is coded in the control of solutions of $P f=-1$. Our analysis of these is based on their intimate relation to the solutions of the Schrödinger equation whose asymptotics are well studied in the case at hand.

The physical background of this is: $P f$ is the on-shell part of the commutator of $H$ and the distortion operator:

$$
\left[\frac{1}{2}(f D+D f), H\right]=-i\left(P f+f^{\prime}(H-E)+(H-E) f^{\prime}\right)
$$


$\left(\left(\mathrm{D}:=-i \partial_{x}\right)\right)$. In this sense $P f=-1$ means that $\frac{1}{2}(f D+D f)(t)$ is decreasing along trajectories: for a wave packet concentrated near the energy shell $\left\langle\psi(t), \frac{1}{2}(f D+\right.$ $D f) \psi(t)\rangle \sim-t$ for $i \partial_{t} \psi(t)=H \psi(t)$. Remark that we discuss the full onshell part of the commutator and not, as it is usually done, its classical limit $2(F x+W-E) f^{\prime}+\left(F+W^{\prime}\right) f$.

In more than one dimension the on-shell part of the above commutator is not a function; this is the main obstacle for an extension of our approach to that case, see also [6].

We shall proceed as follows. In Sect. 2 the resonance theory will be given supposing existence of a suitable distortion field $f$. In Sect. 3 we shall establish our analytic result: the existence of a unique, slowly oscillating $f$ with $\left\|f^{\prime}\right\|_{\infty} \leq e^{c / F}$ and $P f=-1$ on a sufficiently large region and prove Theorem 1 .

Finally a remark concerning notation: the quantities which are derived from $H$ and $f$ depend on the parameters $E, F, \theta$; if we feel that this is clear from the context we shall not burden the reader with an explicit notation of the indices. A generic constant not depending on the parameters will be denoted by cte., the norm of $u \in L^{2}(\mathbf{R})$ by $\|u\| . C_{b}^{\infty}$ are the functions whose derivatives are bounded.

\section{Resonances}

We shall now define resonances by spectral deformation and establish a theory of resonance free domains.

Let $W$ satisfy (A), $F>0$; denote by

$$
H_{F}:=-\Delta+F x+W(x)
$$

-or simply $H$ - the selfadjoint operator which by the Faris-Lavine theorem (see Reed and Simon [24]) is uniquely defined by extension from $C_{0}^{\infty}(\mathbf{R})$.

For $f \in C^{\infty}(\mathbf{R}, \mathbf{R}) \quad \sup _{x \in \mathbf{R}}\left|f^{\prime}(x)\right|<L$, define a family of unitary transformations by

$$
U(\theta) \psi(x):=\left(1+\theta f^{\prime}\right)^{1 / 2} \psi(x+\theta f(x)) \quad\left(\psi \in L^{2}(\mathbf{R}), \quad-1 / L<\theta<1 / L\right) .
$$

A resonance is understood to be a complex discrete eigenvalue of

$$
\begin{gathered}
H(\theta)=U(\theta) H U^{-1}(\theta) \\
=-\left(1+\theta f^{\prime}\right)^{-1 / 2} \nabla\left(1+\theta f^{\prime}\right)^{-1} \nabla\left(1+\theta f^{\prime}\right)^{-1 / 2}+F x+F \theta f(x)+W(x+\theta f(x))
\end{gathered}
$$

analytically Type (A) (see Kato [21]) continued to complex $|\theta|<1 / L$.

There exist meromorphic continuations of sufficiently many matrix elements of the resolvent $(H-E)^{-1}$ to a complex neighborhood of each real energy $E$, whose poles are the eigenvalues of $H(\theta)$. This concept of resonance has a long story which can be traced from Hunziker [19].

We start by proving the analyticity of $H(\theta)$ assuming that an $f$ with the desired properties exists, then continue by showing that certain sets are free of spectrum of $H(\theta)$.

$$
\begin{aligned}
& \text { Denote }\langle x\rangle:=\left(1+F^{2} x^{2}\right)^{1 / 2},\|f\|_{\Omega}:=\sup _{x \in \Omega}|f(x)| \text {, and } \\
& \|\| f\|\|:=\sum_{k=0}^{3}\left\|\langle x\rangle^{\frac{(1-k)}{2}} \partial^{k} f\right\|_{\mathbf{R}^{+}}+\|f\|_{\mathbf{R}^{-}}+\left\|\langle x\rangle f^{\prime}\right\|_{\mathbf{R}^{-}}+\left\|\langle x\rangle^{\frac{1}{2}} f^{\prime \prime}\right\|_{\mathbf{R}^{-}}+\left\|f^{\prime \prime \prime}\right\|_{\mathbf{R}^{-}}
\end{aligned}
$$

We have 
Theorem 2. Let $f \in C^{3}(\mathbf{R})$ such that for some $c>0: \||| f|| \leq e^{c / F}$. Then there exists $c^{\prime}>c, F_{0}>0$ such that for $F \leq F_{0}, H_{F}(\theta)$ can be extended to a Type (A) analytic family in $\left\{\theta \in \mathbf{C} ;|\theta| \leq e^{-c^{\prime} / F}\right\}$.

Proof. Since $C_{0}^{\infty}(\mathbf{R})$ is a core for $H_{F}$ it is sufficient to show equivalence of the graph norms of $H_{F}(\theta)$ for all $|\theta|$ small enough and analyticity of $\theta \mapsto H_{F}(\theta) u$ for $u \in C_{0}^{\infty}(\mathbf{R})$.

Denote $J(x, \theta):=1+\theta f^{\prime}(x)$, by $B$ a generic bounded operator and by cte. a generic positive real number. It holds:

$$
\begin{aligned}
H(\theta)-H= & \nabla g_{1} \nabla-J^{-1 / 2}\left(J^{-1}\left(J^{-1 / 2}\right)^{\prime}\right)^{\prime} \\
& +\theta F f+W(x+\theta f(x))-W(x) \\
= & g_{1} \Delta+g_{1}^{\prime} \nabla+g_{2}+B
\end{aligned}
$$

with $g_{1}:=1-J^{-2}, g_{2}:=-J^{-1 / 2}\left(J^{-1}\left(J^{-1 / 2}\right)^{\prime}\right)^{\prime}$. Let $\chi^{+}$be a real $C^{\infty}$ characteristic function of $(r, \infty)$ for an $r>0, \chi^{-}:=1-\chi^{+}, g^{ \pm}:=\chi^{ \pm} g$ for a function $g$. From the assumption on $f$ it follows with

$$
\begin{gathered}
\left\|g_{1}^{+}\right\|_{\mathbf{R}}+\left\|\langle x\rangle g_{1}^{-}\right\|_{\mathbf{R}}+\left\|\langle x\rangle^{-1 / 2}\left(g_{1}^{\prime}\right)^{+}\right\|_{\mathbf{R}^{+}} \\
\left\|\langle x\rangle^{1 / 2}\left(g_{1}^{\prime}\right)^{-}\right\|_{\mathbf{R}}+\left\|\langle x\rangle^{-1} g_{2}^{+}\right\|_{\mathbf{R}}+\left\|g_{2}^{-}\right\|_{\mathbf{R}} \leq c t e .|\theta| e^{c / F}
\end{gathered}
$$

Combined with the following four estimates (see also below):

$$
\left\|\langle x\rangle^{-1} \Delta u\right\|+\left\|\langle x\rangle^{-1 / 2} D u\right\|+\left\|\chi^{+} \Delta u\right\|+\left\|\chi^{+}\langle x\rangle u\right\| \leq \text { cte. }\left(\left\|H_{F} u\right\|+\|u\|\right),
$$

this gives $\left\|\left(H_{F}(\theta)-H_{F}\right) u\right\| \leq$ cte. $|\theta| e^{c / F}\left(\left\|H_{F} u\right\|+\|u\|\right)$ and so there is a $c^{\prime}>0$ and an $\alpha<1$ such that for $|\theta| \leq e^{-c^{\prime} / F}$,

$$
\left\|\left(H_{F}(\theta)-H_{F}\right) u\right\| \leq \alpha\left(\left\|H_{F} u\right\|+\|u\|\right),
$$

which implies equivalence of the $H_{F}(\theta)$ graph-norms. Analyticity of $\theta \mapsto H_{F}(\theta) u$ is immediate. We finish the proof giving one of the operator estimates used above:

$$
\begin{aligned}
\left\|\langle x\rangle^{-1 / 2} D u\right\|^{2} & =-\left\langle u, \nabla\langle x\rangle^{-1} \nabla u\right\rangle . \\
& =-\frac{1}{2}\left\langle u,\left(\langle x\rangle^{-1} \Delta+\Delta\langle x\rangle^{-1}\right) u\right\rangle+\frac{1}{2}\left\langle u,\left(\langle x\rangle^{-1}\right)^{\prime \prime} u\right\rangle \\
& \leq \frac{1}{2} \mid\left\langle u,\langle x\rangle^{-1} H_{F}+H_{F}\langle x\rangle^{-1}\langle u\rangle|+|\langle u, B u\rangle\right| \\
& \leq \text { cte. }\left(\left\|\langle x\rangle^{-1} u\right\|\left\|H_{F} u\right\|+\|u\|^{2}\right) \leq \text { cte. }\left(\left\|H_{F}\right\|^{2}+\|u\|^{2}\right) .
\end{aligned}
$$

We are now coming to the announced theory of resonance free domains: Technically the simple idea to prove absence of resonances explained in the introduction does not work for large values of $x$ where we shall use ellipticity instead. We introduce a family of helper functions which satisfy the following assumptions:

(T) For $F>0$ let $a \in C_{b}^{\infty}(\mathbf{R}, \mathbf{R}), a(x) \in[-1,0]$,

$$
\begin{aligned}
& a(x)=-1 \quad\left(x \in\left(-\infty,\left(1+\|W\|_{\mathbf{R}}+E\right) / F\right)\right), \\
& a(x)=0 \quad\left(x \in\left(\left(2+\|W\|_{\mathbf{R}}+E\right) / F, \infty\right)\right) .
\end{aligned}
$$

The result is: 
Theorem 3. Assume (T) holds for a. Let $f$ be a solution of $P f=a$ such that $\exists c>$ $0 \forall E \in \mathbf{R}:\left|\|f \mid\| \leq e^{c / F}\right.$. Define b by $a+b=-1$.Then

there exists $c^{\prime}>2 c, F^{\prime}>0$ such that for $F \leq F^{\prime}$ it holds for $|\theta|<e^{-c^{\prime} / F}$ and $\operatorname{Im} \theta=\beta \geq 0$ :

$$
\bigcup_{E \in \mathbf{R}}\left\{z \in \mathbf{C} ; \inf _{x \in \mathbf{R}} \operatorname{Im}\left(\left((1+i \beta b)\left(1+i \beta f^{\prime}\right)\right)^{2}(z-E)\right)>-\frac{\beta}{2}\right\} \subset \rho\left(H_{F}(\theta)\right)
$$

where $\rho$ denotes the resolvent set.

Proof. A real shift in $\theta$ leads to a unitary equivalent $H(\theta)$, so it is sufficient to treat the case $\theta=i \beta$. Because of technicalities we treat the region of ellipticity separately, this is accounted for by the function $b$. Denote $\mu(\theta):=(1+\theta b)\left(1+\theta f^{\prime}\right)$. Let $E \in \mathbf{R}$. It is known (cf.: $[6,7]$, see also [8]) that

$$
\left\{z \in \mathbf{C} ; \operatorname{Im}\langle u, \mu(\theta)(H(\theta)-z) \mu(\theta) u\rangle \leq 0 \quad\left(u \in C_{0}^{\infty}(\mathbf{R})\right\} \subset \rho\left(H_{F}(\theta)\right)\right.
$$

So all we have to do in order to prove the assertion is to show

$$
\operatorname{Im}(\mu(\theta)(H(\theta)-E) \mu(\theta)) \leq-\beta / 2
$$

in the quadratic form sense on $C_{0}^{\infty}(\mathbf{R})$.

Denoting $V(x):=F x+W(x)-E, J(x, \theta):=\left(1+\theta f^{\prime}(x)\right)$ and using the identity $g \nabla h \nabla g=\nabla g^{2} h \nabla+g\left(h g^{\prime}\right)^{\prime}$ we have

$$
(\mu(H-E) \mu)(\theta)=(1+\theta b)\left(-\Delta+J^{2} V(x+\theta f)-\frac{1}{2} J^{1 / 2}\left(J^{-3 / 2} J^{\prime}\right)^{\prime}\right)(1+\theta b) .
$$

Using $b<0$ this implies

$\operatorname{Im}(\mu(H-E) \mu)(\theta) \leq \operatorname{Im}\left(\mu^{2} V(x+\theta f)-\theta b^{\prime \prime}(1+\theta b)-(1+\theta b)^{2}\left(\frac{1}{2 J} J^{\prime \prime}-\frac{3}{4 J^{2}} J^{\prime 2}\right)\right)$.

A calculation using Taylor expansion in $\theta$ shows that the right-hand side of this inequality is

$$
\operatorname{Im}\left(\theta\left(P f+2 b V+b^{\prime \prime}(1+\theta b)+\theta\left(O(1)+O(1) f^{\prime \prime 2}+O(1) f^{\prime \prime \prime} f^{\prime}\right)\right)\right)
$$

where, using $\|f\|+\left\|f^{\prime}\right\| \leq e^{c / F}$, one sees that $\theta O(1)$ is uniformly bounded in $F, x$ for $\theta<e^{-2 c / F}$. By the assumption on $f$ we have $\left|f^{\prime \prime 2}(x)+f^{\prime \prime \prime} f^{\prime}(x)\right| \leq e^{2 c / F}(b V(x)+1)$. Using $b^{\prime \prime}=O\left(F^{2}\right)$ we see: for any $1>\epsilon>0$ there exists $c^{\prime}>2 c, F^{\prime}>0$ such that for $F \leq F^{\prime}$ it holds for $0 \leq|\theta|<e^{-c^{\prime} / F}$ :

$$
\operatorname{Im}(\mu(H-E) \mu)(\theta) \leq \beta(P f+(2-\epsilon) b V+\epsilon) \leq \beta(a+b+\epsilon)<\beta(-1+\epsilon) .
$$




\section{The Distortion Field}

In this section we shall establish existence of the function $f$ needed for the machinery of Sect. 2. As a corollary we shall give the proof of Theorem 1.

Let $a$ be a smooth negative switching function which is zero for large $x$ and -1 to the left of the first turning point of $-\Delta+F x+W(x)-E$. Denote again $V(x):=F x+W(x)-E$.

We shall construct the unique solution of the equation

$$
P f=\left(-\partial^{3} / 2+V \partial+\partial V\right) f=a
$$

subject to the growth condition

$$
|| f \mid \| \leq e^{c / F} .
$$

For the construction we use the following nice observation: Let $\varphi_{1}, \varphi_{2}$ be a fundamental system of the time-independent Schrödinger equation

$$
-\Delta \varphi+V \varphi=0,
$$

then the products $\varphi_{1}^{2}, \varphi_{2}^{2}, \varphi_{1} \varphi_{2}$ form a fundamental system of

$$
P g=0 .
$$

This relation is well known for the Airy equation, in general we could trace it to McKean and Trubowitz [22]. We thank E. Mourre and V. Ovsienko for telling us about it. We refer also to Gelfand and Zakharevich [12] who studied $P f=0$ as a spectral problem on the circle for periodic $V$.

Let us describe why it is non-trivial to gain control on the derivatives of $f$ and how we do it. From the standard asymptotic approximation of solutions of second order differential equations [23] one has that a generic solution of the homogeneous equation $P g=0$ behaves like

$$
\begin{gathered}
g(x) \sim_{x \rightarrow \infty} \frac{1}{\sqrt{V}}\left(\alpha e^{2 \int^{x} \sqrt{V}}+\beta e^{-2 \int^{x} \sqrt{V}}+\gamma\right), \\
g(x) \sim_{x \rightarrow-\infty} \frac{1}{\sqrt{|V|}}\left(\alpha_{l} \cos \left(2 \int^{x} \sqrt{V}\right)+\beta_{l} \sin \left(2 \int^{x} \sqrt{V}\right)+\gamma_{l}\right)
\end{gathered}
$$

with some $\alpha, \beta, \gamma, \alpha_{l}, \beta_{l}, \gamma_{l} \in \mathbf{C}$.

We see that generic $f$ with $P f=a$ will exhibit exponential growth to $\infty$ and fast oscillations to $-\infty$. It turns out that the inconvenient terms are killed if the Sturm-Liouville type boundary condition

$$
(V \partial+\partial V) f(x)-a(x) \rightarrow_{x \rightarrow \pm \infty} 0
$$

is imposed.

To have a flavour of this, note that smooth solutions of the classical equation $(V \partial+\partial V) h=0$ behave like

$$
h(x) \sim_{|x| \rightarrow \infty} \frac{c}{\sqrt{|V|(x)}} .
$$

Because of the presence of turning points in a region which scales like $1 / F$ these exist, however, only for large $|x|$.

The idea of imposing this boundary condition is implemented in the construction of the Green's function for the problem: 
Theorem 4. Let $E \in \mathbf{R}, F>0, W \in C_{b}^{\infty}(\mathbf{R}, \mathbf{R})$. Denote $V(x):=F x+W(x)-E$. There exists a function $G \in C^{\infty}\left(\mathbf{R}^{2} \backslash\{x=y\}, \mathbf{R}\right) \cap C^{1}\left(\mathbf{R}^{2}, \mathbf{R}\right)$ such that for $a \in C_{b}^{\infty}(\mathbf{R}, \mathbf{R}), a(x)=0 \quad(x>r>0$ suitable $)$ the function

$$
f(x):=\int_{\mathbf{R}} G(x, y) a(y) d y \quad(x \in \mathbf{R})
$$

is in $C^{\infty}(\mathbf{R}, \mathbf{R})$ and is the unique solution of

$$
P f=\left(-\partial^{3} / 2+V \partial+\partial V\right) f=a
$$

such that $\exists c>0 \forall F>0$

$$
\|\| f \| \leq e^{c / F}
$$

where

$$
\|\| f\|\|:=\sum_{k=0}^{3}\left\|\langle x\rangle^{\frac{(1-k)}{2}} \partial^{k} f\right\|_{\mathbf{R}^{+}}+\|f\|_{\mathbf{R}^{-}}+\left\|\langle x\rangle f^{\prime}\right\|_{\mathbf{R}^{-}}+\left\|\langle x\rangle^{\frac{1}{2}} f^{\prime \prime}\right\|_{\mathbf{R}^{-}}+\left\|f^{\prime \prime \prime}\right\|_{\mathbf{R}^{-}}
$$

Furthermore, $c$ can be chosen uniformly in $E$.

Proof. We shall give an explicit construction of the Green's function in terms of solutions of (Sch), then verify the assertions.

Let us establish the rules of the game:

Lemma 1. There exist two real, independent solutions $\varphi_{+}, \varphi_{-} \in C^{\infty}$ of (Sch) with the following properties: Choose $x_{l}<x_{r}$ such that $|V(x)|>1 \quad\left(x \notin\left(x_{l}, x_{r}\right)\right)$ and $\left|V(x)+V^{\prime \prime} / V(x)\right|>1 \quad\left(x \in\left(-\infty, x_{l}\right)\right)$, define $s(x):=\left|V(x)+V^{\prime \prime} / V(x)\right|^{1 / 2} \quad\left(x \in\left(-\infty, x_{l}\right)\right)$. Then

(1) There exist $\alpha_{+}, \alpha_{-} \in \mathbf{C} \backslash(0) ; r_{+}, r_{-}, l \in C^{\infty}(\mathbf{R}), c>0$ such that for $F>0$ :

$$
\begin{gathered}
\varphi_{ \pm}(x)=\frac{1}{V(x)^{1 / 4}} \exp \left( \pm \int_{x_{r}}^{x} \sqrt{V}\right)\left(1+r_{ \pm}(x)\right) \quad\left(x>x_{r}\right), \\
\varphi_{ \pm}(x)=\frac{1}{s(x)^{1 / 2}}\left(\alpha_{ \pm} \exp \left(i \int_{x_{l}}^{x} s\right)+\overline{\alpha_{ \pm}} \exp \left(-i \int_{x_{l}}^{x} s\right)\right)(1+l(x)) \quad\left(x<x_{l}\right), \\
\text { Wronskian }\left(\varphi_{+}, \varphi_{-}\right)=2=2 i\left(\alpha_{+} \overline{\alpha_{-}}-\overline{\alpha_{+}} \alpha_{-}\right) .
\end{gathered}
$$

Furthermore for $I_{r}:=\left(x_{r}, \infty\right), I_{l}:=\left(-\infty, x_{l}\right)$ it holds:

$$
\sum_{k=0}^{2}\left(\left\|\langle x\rangle^{-k / 2} \partial^{k} r_{+}\right\| I_{r}+\left\|\langle x\rangle^{(1-k) / 2} \partial^{k} r_{-}\right\|_{I_{r}}+\left\|\langle x\rangle^{(3-k) / 2} \partial^{k} l\right\|_{I_{l}}\right) \leq e^{c / F}
$$

(2) $\exists c>0, \forall F>0$ :

$$
\sum_{k=0}^{1}\left(\left\|\partial^{k} \varphi_{-}^{2}\right\|_{\mathbf{R}}+\left\|\partial^{k} \varphi_{+}^{2}\right\|_{\left(-\infty, x_{r}\right)}\right)+\left\|\partial^{2} \varphi_{ \pm}^{2}\right\|_{\left(x_{l}, x_{r}\right)} \leq e^{c / F}
$$

For $g \in C_{b}^{2}$ it holds:

(3) for $x_{r}<y<x$, 


$$
\begin{gathered}
\left|\int_{y}^{x} \frac{g}{\sqrt{V}}(z) \exp \left(-2 \int_{x_{r}}^{z} \sqrt{V}\right) d z+\left(\frac{g}{2 V}(z) \exp \left(-2 \int_{x_{r}}^{z} \sqrt{V}\right)\right)\right|_{y}^{z=x} \mid \\
\leq \exp \left(-2 \int_{x_{r}}^{y} \sqrt{V}\right) \int_{y}^{x}\left(\left|\frac{g^{\prime}}{2 V}\right|+\left|\frac{g V^{\prime}}{V^{2}}\right|\right)
\end{gathered}
$$

(4) for $y<x<x_{l}$,

$$
\begin{gathered}
\left|\int_{y}^{x} \frac{g}{s}(z) \exp \left(2 i \int_{z}^{x} s\right) d z-\left(\frac{g}{2 i s^{2}}+\frac{g^{\prime}}{4 s^{3}}+\frac{g s^{\prime}}{2 s^{4}}\right)(z) \exp \left(2 i \int_{z}^{x} s\right)\right|_{z=y}^{z=x} \mid \\
\leq \text { cte. } \int_{y}^{x}\left(\left|\frac{g^{\prime \prime}}{s^{3}}\right|+\left|\frac{g s^{\prime \prime}}{s^{4}}\right|+\left|\frac{g s^{\prime 2}}{s^{5}}\right|\right) ;
\end{gathered}
$$

(5) for $x_{r}<y<x$ or $y<x<x_{l}$,

$$
\int_{y}^{x} \frac{g}{\sqrt{|V|}} \leq\left.\frac{c t e}{F}\|g\||V|^{1 / 2}(z)\right|_{z=y} ^{z=x}
$$

Proof of Lemma 1.

(1) Cf.: [23].

(2) From Gronwall's inequality it holds for solutions $\varphi$ of (Sch):

$$
|\varphi(x)|+\left|\varphi^{\prime}(x)\right| \leq\left(\left|\varphi\left(x_{0}\right)\right|+\left|\varphi^{\prime}\left(x_{0}\right)\right|\right) \exp \left((1+\|W\|)\left(x-x_{0}\right)+F / 2\left(x-x_{0}\right)^{2}\right) .
$$

The assertion follows from the asymptotics in the region $\left(x_{r}, \infty\right)$, from the estimate in the region $\left(x_{l}, x_{r}\right)$, again from the asymptotics in $\left(-\infty, x_{l}\right)$.

$(3,4,5)$ are by integration by parts. This finishes the proof of Lemma 1 .

We resume the proof of Theorem 4: Take $\varphi_{+}, \varphi_{-}$as in the Lemma 1.

\section{Uniqueness:}

Let $f$ be a solution with \|\|$f \| \mid<\infty$. An arbitrary solution $h$ is given by

$$
h=c_{+} \varphi_{+}^{2}+c_{-} \varphi_{-}^{2}+c_{+-} \varphi_{+} \varphi_{-}+f .
$$

||$|h|||<\infty$ implies absence of growth and oscillations:

$$
c_{+}=0,\left(\begin{array}{cc}
\alpha_{-}^{2} & \alpha_{+} \alpha_{-} \\
\bar{\alpha}_{-}^{2} & \bar{\alpha}_{+} \overline{\bar{\alpha}}_{-}
\end{array}\right)\left(\begin{array}{c}
c_{-} \\
c_{+-}
\end{array}\right)=0 \text {. With }\left|\begin{array}{cc}
\alpha_{-}^{2} & \alpha_{+} \alpha_{-} \\
\bar{\alpha}_{-}^{2} & \bar{\alpha}_{+} \bar{\alpha}_{-}
\end{array}\right|=i\left|\alpha_{-}\right|^{2} \neq 0 .
$$

It follows $c_{+}=c_{-}=c_{+-}=0$, so $h=f$.

\section{Existence:}

We give $G$ constructively, then verify the properties:

$$
G(x, y)=
$$

$$
\left\{\begin{array}{l}
\frac{1}{4}\left(\varphi_{+}^{2}+\left|\frac{\alpha_{+}}{\alpha_{-}}\right|^{2} \varphi_{-}^{2}-2 R e \frac{\alpha_{+}}{\alpha_{-}} \varphi_{+} \varphi_{-}\right)(x) \varphi_{-}^{2}(y) \quad(x \leq y) \\
\frac{1}{2} \varphi_{+} \varphi_{-}(x)\left(\varphi_{+} \varphi_{-}-R e \frac{\alpha_{+}}{\alpha_{-}} \varphi_{-}^{2}\right)(y)-\frac{1}{4} \varphi_{-}^{2}(x)\left(\varphi_{+}^{2}-\left|\frac{\alpha_{+}}{\alpha_{-}}\right|^{2} \varphi_{-}^{2}\right)(y) \quad(x \geq y)
\end{array}\right.
$$

The solutions of (Sch) are $C^{\infty}$. It is algebraic to verify:

$$
G \in C^{\infty}\left(\mathbf{R}^{2} \backslash\{x=y\}\right) \cap C^{1}\left(\mathbf{R}^{2}\right), \quad P^{x} G(x, y)=0 \quad(x \neq y),
$$




$$
-\frac{1}{2}\left(\left(\partial_{x}^{2} G\right)(x, x-0)-\left(\partial_{x}^{2} G\right)(x, x+0)\right)=1 \quad(x \in \mathbf{R}) .
$$

Thus $f(x)=\int_{\mathbf{R}} G(x, y) a(y) d y$ is a solution of $P f=a$; the existence of the oscillatory integral will follow from the estimates which we readily shall prove using Lemma 1. Define $h, g_{1}, g_{2}, f_{u}, f_{d}$ by

$$
G(x, y)=:\left\{\begin{array}{l}
h(x) \varphi_{-}^{2}(y) \quad(x \leq y) \\
\varphi_{+} \varphi_{-}(x) g_{1}(y)+\varphi_{-}^{2}(x) g_{2}(y) \quad(x \geq y)
\end{array}\right.
$$

$f=f_{u}+f_{d}$ with

$$
\begin{aligned}
f_{u}(x) & :=h(x) \int_{x}^{\infty} \varphi_{-}^{2} a \\
f_{d}(x) & :=\varphi_{+} \varphi_{-}(x) \int_{-\infty}^{x} g_{1} a+\varphi_{-}^{2}(x) \int_{-\infty}^{x} g_{2} a .
\end{aligned}
$$

In the region $I_{l}=\left(-\infty, x_{l}\right)$ it holds $P h=0$ and $h=\frac{1}{\left|\alpha_{-}\right|^{2}}\left|V(x)+V^{\prime \prime} / V(x)\right|^{-1 / 2}(1+l)^{2}$ so

$$
\left\|\langle x\rangle^{1 / 2} h\right\|_{I_{l}}+\sum_{k=1}^{3}\left\|\langle x\rangle^{(4-k) / 2} \partial^{k} h\right\|_{I_{l}} \leq e^{c / F} .
$$

It follows from Lemma 1, writing $\int_{x}^{\infty} \varphi_{-}^{2} a=\left(\int_{x}^{x_{r}}+\int_{x_{r}}^{\infty}+\int_{x_{l}}^{x_{r}}\right) \varphi_{-}^{2} a$ :

$$
\sum_{k=1}^{3}\left\|\langle x\rangle^{(3-k) / 2} \partial^{k} f_{u}\right\|_{I_{l}}+\left\|f_{u}\right\|_{I_{l}} \leq e^{c / F}\left(\sum_{k=0}^{2}\left\|\partial^{k} a\right\| \times \sum_{k=1}^{4}\left\|\partial^{k} V\right\|\right) .
$$

Concerning $f_{d}$ we have by a similar argument:

$$
\sum_{k=0}^{3}\left\|\langle x\rangle^{(1-k) / 2} \partial^{k}\left(\varphi_{+} \varphi_{-}\right)\right\|_{I_{l}}+\sum_{k=0}^{3}\left\|\langle x\rangle^{(1-k) / 2} \partial^{k} \varphi_{-}^{2}\right\|_{I_{l}} \leq e^{c / F} .
$$

A calculation shows that $g_{1}, g_{2}$ have only oscillating components (sic!) so $\left\|\langle x\rangle \int_{-\infty}^{x} g_{1 ; 2} a\right\|_{I_{l}} \leq e^{c / F}$ and

$$
\left\|f_{d}\right\|_{I_{l}}+\sum_{k=1}^{3}\left\|\langle x\rangle^{(3-k) / 2} \partial^{k} f_{d}\right\|_{I_{l}} \leq e^{c / F}
$$

In the region $I_{r}=\left(x_{r}, \infty\right)$ it holds

$$
\begin{gathered}
\sum_{k=0}^{3}\left\|e^{-2 \int^{x} \sqrt{V}}\langle x\rangle^{(1-k) / 2} \partial^{k} h\right\|_{I_{r}} \leq e^{c / F}, \\
\int_{x}^{\infty} \varphi_{-}^{2} a \leq e^{c / F} e^{-2 \int^{x} \sqrt{V}}\langle x\rangle^{-1}\left(1+\left\|\langle x\rangle a^{\prime}\right\|_{\mathbf{R}_{+}}\right),
\end{gathered}
$$

which gives the estimate 


$$
\sum_{k=0}^{3}\left\|\langle x\rangle^{(3-k) / 2} \partial^{k} f_{u}\right\|_{I_{r}} \leq e^{c / F} .
$$

Concerning $f_{d}: \varphi_{+} \varphi_{-}=\frac{1}{\sqrt{V}}\left(1+r_{+}\right)\left(1+r_{-}\right)$, so

$$
\begin{gathered}
\sum_{k=0}^{3}\left\|\langle x\rangle^{(1-k) / 2} \partial^{k}\left(\varphi_{+} \varphi_{-}\right)\right\|_{I_{r}}+\sum_{k=0}^{3}\left\|\langle x\rangle^{(1-k) / 2} e^{2 \int^{x} \sqrt{V}} \partial^{k} \varphi_{-}^{2}\right\|_{I_{r}} \leq e^{c / F} \\
\int_{-\infty}^{x} g_{1 ; 2} a \leq \int_{-\infty}^{r} g_{1 ; 2} a \leq e^{c / F} \text { gives } \\
\sum_{k=0}^{3}\left\|\langle x\rangle^{(1-k) / 2} \partial^{k} f_{d}\right\|_{\mathbf{R}_{+}} \leq e^{c / F} .
\end{gathered}
$$

In the region $\left(x_{l}, x_{r}\right)$ the estimates are straightforward. Thus \|\|$f \| \mid \leq e^{c / F}$ and we have finished the proof of Theorem 4.

This finishes the construction of the distortion function $f$ which is needed as input to the resonance theory of Sect. 2 . We end with the

Proof of Theorem 1. By Theorem 4 there exists $f \in C^{\infty}$ with $\|\mid f\| \| \leq e^{c / F}$ and $P f=a$ for $a$ satisfying (T). By Theorem $2 H_{F}(\theta)$ is analytic for $|\theta|<e^{-c / F}$. As there exists $c>0, F^{\prime}>0$ such that

$$
\begin{aligned}
& \left\{z \in \mathbf{C} ; \operatorname{Im} z \geq e^{-c / F}\right\} \subset \\
& \bigcup_{E \in \mathbf{R}}\left\{z \in \mathbf{C} ; \inf _{x \in \mathbf{R}} \operatorname{Im}\left(\left((1+i \beta b)\left(1+i \beta f^{\prime}\right)\right)^{2}(z-E)\right)>-\frac{\beta}{2}\right\},
\end{aligned}
$$

Theorem 3 implies absence of resonances in

$$
\left\{E \in \mathbf{C} ; \operatorname{Im} E \geq e^{-c / F}\right\} .
$$

\section{References}

1. Agler, J. and Froese, R.: Existence of Stark ladder resonances. Commun. Math. Phys. 100, 161-171 (1985)

2. Ahia, F.: Lower bounds on width of Stark resonances in one dimension. Lett. Math. Phys. 24, 21-29 (1992)

3. Avron, J.E.: The lifetime of Wannier ladder states. Ann. Phys. 143, 33-53 (1982)

4. Bentosela, F., Carmona, R., Duclos, P., Simon, B., and Souillard, B.: Schrödinger operators with an electric field and random or deterministic potentials. Commun. Math. Phys. 88, 387-397 (1983)

5. Bentosela, F. and Grecchi,V.: Stark Wannier ladders. Commun. Math. Phys. 142, 169-192 (1991)

6. Briet, P.: Estimates on distorted resolvents and application to Stark hamiltonians. Rev. Math. Phys. to appear (1996)

7. Briet, P., Combes, J.M., and Duclos,P.: On the location of resonances for Schrödinger operators in the semi classical limit 1: Free domains of resonances. J. Math. Anal.Appl. 126, 90-99 (1987)

8. Briet, P., Combes, J.M., and Duclos,P.: Spectral stability under tunneling. Commun. Math. Phys. 126, 133-156 (1989)

9. Buslaev,V.S. and Dmitrieva, L.A.: A Bloch electron in an external field. Leningrad Math. J. 1(2), 287-320 (1990) 
10. Combes, J.M., and Hislop,P.: Stark ladder resonances for small electric fields. Commun. Math. Phys. 140, 291-320 (1991)

11. Fernandez, C., and Lavine, R.: Lower bounds for resonance widths in potential and obstacle scattering. Commun. Math. Phys. 128(2), 263-284 (1990)

12. Gelfand,I.M., and Zakharevich,I.: The spectral theory for a pencil of skewsymmetrical operators of the third order. Comm. Pure. Appl. Math. 157, 1031-1041 (1994)

13. Grecchi, V., Maioli, M., and Sacchetti,A.: Stark resonances in disordered systems. Commun. Math. Phys. 146, 231-240 (1992)

14. Grecchi, V., Maioli, M., and Sacchetti, A.: Stark ladders of resonances: Wannier ladders and perturbation theory. Commun. Math. Phys. 159, 605-618 (1994)

15. Grecchi,V., and Sacchetti, A.: Crossing and anticrossing of resonances: The Wannier-Stark ladders. Ann. Phys. 241, 258-284 (1995)

16. Harrel II, E.M.: General lower bounds for resonances in one dimension. Commun. Math. Phys. 86, 221-225 (1982)

17. Helffer, B., and Sjöstrand, J.: Résonances en limite semi-classique. Bull. Soc. Math. France 114(3), No.24/25 (1986)

18. Herbst,I.W., and Howland, J.S.: The Stark ladder and other one-dimensional external field problems. Commun. Math. Phys. 80, 23-42 (1981)

19. Hunziker, W.: Distortion analyticity and molecular resonance curves. Ann. Inst. Henri Poincaré 43(4), 339-358 (1986)

20. Jensen, A.: Bounds on resonances for Stark-Wannier and related hamiltonians. J. Oper. Theory 19(1), 69-80 (1988)

21. Kato, T.: Perturbation Theory for Linear Operators. Berlin-Heidelberg-NewYork: Springer (1980)

22. McKean, H.P., and Trubowitz, E.: Hill's operator and hyperelliptic function theory in the presence of infinitely many branch points. Comm. Pure. Appl. Math. 29(1), 143-226 (1976)

23. Olver, F.W.J.: Asymptotics and Special Functions. London-NewYork: Academic Press (1974)

24. Reed, M., and Simon, B.: Methods of Modern Mathematical Physics II. London-NewYork: Academic (1975)

25. Wannier, G.H.: Wave functions and effective hamiltonians for Bloch electrons in an electric field. Phys. Rev. 117, 432-439 (1960)

\section{Communicated by B. Simon}

This article was processed by the author using the $\mathrm{LAT}_{\mathrm{E}} \mathrm{X}$ style file pljour1 from Springer-Verlag. 
